PROCEEDINGS OF THE

AMERICAN MATHEMATICAL SOCIETY

Volume 48, Number 1, March 1975

\title{
AUTOMORPHISMS COMPATIBLE WITH GROUP ACTIONS
}

\section{MICHAEL A. GAUGER}

ABSTRACT. Let $A$ be a group of bijections of the set $\theta$ and let $G$ be a subgroup of $A$. Our purpose here is to introduce several other subgroups of $A$ consisting of bijections compatible with the $G$-orbit structure on $\vartheta$ and to investigate these groups when $\vartheta$ is the Grassmann variety of all $p$-dimensional subspaces of a vector space $V$ and $G$ is a group of automorphisms of the Grassmannian induced by a group of linear transformations on $V$.

1. Introduction and preliminaries. Let $A$ be a group of bijections of the set $C$ and let $G$ be a subgroup of $A$. Our purpose here is to introduce several other subgroups of $A$ consisting of bijections compatible with the $G$-orbit structure on $\mathcal{C}$, and to investigate these groups when $\mathcal{C}$ is the Grassmann variety of all $p$-dimensional subspaces of a vector space $V$ and $G$ is a group of automorphisms of the Grassmannian induced by a group of linear transformations on $V$. The problem just mentioned is connected with the determination of duality theories for metabelian Lie algebras $[3, \S 31$.

If $G$ and $A$ are as above, the group $\mathcal{F}$ consisting of ele ments of $A$ fixing each $G$-orbit is called the orbit fixing group of $G$ in $A$. The subgroup $\mathcal{S}$ of $A$ consisting of all elements of $A$ taking $G$-orbits to $G$-orbits is called the orbit shuffling group of $G$ in $A$. Then $G \subseteq \mathcal{F} \subseteq \mathcal{S} \subseteq A$, and $\mathcal{S}$ consists

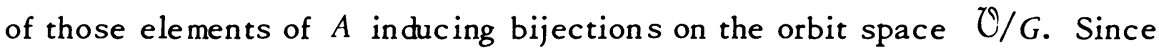
$\mathcal{F}$ is the kernel of the natural map $\mathcal{S} \rightarrow \mathrm{Bij}(\mathcal{O} / G)$, we see that $\mathcal{F}$ is normal in $\mathcal{S}$. The main result achieved here is the

Theorem. Suppose $G$ is a connected, irreducible algebraic subgroup of $\mathrm{GL}(V)$ containing the nonzero scalars. Let $\mathcal{S}, \mathcal{F}$ be the orbit shuffling and fixing groups for the induced action of $G$ on the projective Grassmann variety of all p-dimensional subspaces of $V$. Then $\delta / \mathcal{F}$ is a finite group.

This result indicates that the behavior observed in [3] is part of a more general phenomena.

Suppose that $\mathcal{O}$ is a set $w$ ith an equivalence relation, and there is a group $G$ acting on $C \mathcal{}$ whose orbits coincide with equivalence classes. We 
avoid mentioning what the group $A$ should be. What choice one makes usually depends on what structure $\mathcal{O}$ might have in addition to being a set. For example, if $\mathcal{O}$ were a variety, $G$ an algebraic group and $C^{\Omega} \times G \rightarrow \mathcal{C}^{n}$ a morphism, one might choose $A$ to be the "algebraic" automorphisms of $\mathcal{C}$. A typical problem is to find a "canonical form" for elements of O-a "canonical" member of each equivalence class (orbit). Were $\mathcal{F}$ bigger than $G$ it would afford one more transformation to manipulate elements of $\mathcal{O}$ and it could possibly simplify the problem.

$\mathcal{S}$ by itself is apparently not as significant. It is a simple observation, however, that $\mathcal{S} / \mathcal{F}$ is isomorphic to the group of bijections on $\mathcal{O} / G$ induced by $\mathcal{S}$. In connection with the duality theory problem mentioned above, the group $\mathcal{S} / \mathcal{F}$ is in bijective correspondence with the collection of all duality theories satisfying certain axioms (see $\left[3, \S_{1}\right]$ ).

Proposition 1. Let $A$ be a group of bijections of the set $\mathcal{C}$ and let $G$ be a subgroup of $A$. Let $\mathcal{F}$ and $\mathcal{S}$ be the orbit fixing and shuffling groups respectively. Then $\mathcal{S}$ is the normalizer of $\mathcal{F}$ in $A$.

Proof. We have seen already that $\mathcal{F}$ is normal in $\mathcal{S}$. Suppose $\tau$ in $A$ normalizes $\mathcal{F}$. Let $v_{1}, v_{2}$ be in $\mathcal{C}$ and suppose $\theta$ in $G$ satisfies $v_{1} \theta=v_{2}$. Then $v_{2} \tau=v_{1} \theta \tau=v_{1} \tau \theta^{\prime}$ for some $\theta^{\prime}$ in $\mathfrak{F}$. But $\theta^{\prime}$ fixes $G$-orbits so there is a $\theta^{\prime \prime}$ in $G$ with $v_{1} \tau \theta^{\prime \prime}=v_{1} \tau \theta^{\prime}=v_{2} \tau$. Thus $\tau$ shuffles $G$-orbits.

2. Automorphisms compatible with group actions on Grassmann varieties. Let $K$ be an algebraically closed field of characteristic zero and let $V$ be a finite-dimensional $K$-vector space. Suppose $G$ is a connected algebraic subgroup of $\mathrm{GL}(V)$ containing the nonzero scalars. For any $p \leq \operatorname{dim} V$ there is an induced action of $G$ on $G_{p}$-the projective Grassmann variety of $p$ dimensional subspaces of $V \cdot G_{p}$ is the projectivization of the homogeneous affine subvariety $D_{p}$ of $\Lambda^{p} V$ consisting of all decomposable $p$-vectors. Consider the diagram

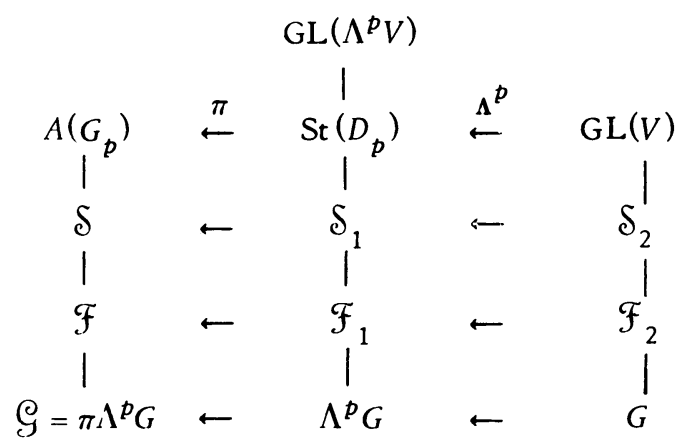


where $A\left(G_{p}\right)$ is the group of "algebraic" automorphisms of $G_{p}, \operatorname{St}\left(D_{p}\right)$ is the stabilizer of $D_{p}$ in $\mathrm{GL}\left(\Lambda^{p} V\right), \pi$ is the natural map, and $\Lambda^{p}$ is the representation of $\mathrm{GL}(V)$ on $\Lambda^{p} V$ given on decomposables by

$$
\Lambda^{p}(\theta)\left(v_{1} \Lambda \cdots \Lambda v_{p}\right)=\theta\left(v_{1}\right) \Lambda \cdots \Lambda \theta\left(v_{p}\right) .
$$

In addition, $\mathcal{S}$ and $\mathcal{F}$ are the orbit shuffling and fixing groups of $\mathcal{S}$ in $A\left(G_{p}\right)$, $\mathcal{S}_{1}=\pi^{-1}(\mathcal{S}), \mathcal{F}_{1}=\pi^{-1}(\mathcal{F}), \mathcal{S}_{2}=\left(\Lambda^{p}\right)^{-1}\left(\mathcal{S}_{1}\right)$, and $\mathcal{F}_{2}=\left(\Lambda^{p}\right)^{-1}\left(\mathcal{F}_{1}\right)$. See $[2$, $\S \S 1,2]$ and $\left[3, \S_{1}\right]$ for further details.

In $[2, \$ 2]$ it is shown that $\pi$ is surjective and the kernel of $\pi$ consists of nonzero scalars. According to Westwick's theorem [4, p. 1127], $\mathrm{St}\left(D_{p}\right)=$ $\Lambda^{p}(\mathrm{GL}(V))$ except when $\operatorname{dim} V / 2=p$, and in this case $\left(\mathrm{St}_{t}\left(D_{p}\right): \Lambda^{p}(\mathrm{GL}(V))\right)=2$.

For the purpose of using Lie algebras later on, we indicate here that all the groups in the diagram are algebraic groups. Since $K$ is algebraically closed, $\Lambda^{p} G$ and $\mathcal{G}$ are algebraic as images of the group $G$ by rational homomorphisms. Let $\tau$ denote the natural map from $D_{p}$ to $G_{p}$. The formula $\Lambda^{p}\left(\alpha I_{V}\right)=\alpha^{p} I_{\Lambda^{p}}$, and the fact that $K$ is algebraically closed shows that $\Lambda^{p}(G)$ also contains the nonzero scalars. Thus if $v$ is a nonzero decomposable $p$-vector the projectivization of the orbit of $v$ by $\Lambda^{p}(G)$ is the orbit of $r(v)$, and conversely, the inverse image under $\tau$ of the orbit of $r(v)$ is the orbit of $v$ by $\Lambda^{p}(G)$. Thus $\mathcal{F}_{1}$ and $\mathcal{S}_{1}$ are the orbit fixing and shuffling groups of $\Lambda^{p}(G)$ in $\operatorname{St}\left(D_{p}\right)$.

Whenever $G$ is a connected algebraic group acting on a variety $C$ by a morphism $\mathcal{O} \times G \rightarrow \mathcal{O}$, orbits are locally closed subvarieties [1, p. 98]. Moreover, the closure of a nonclosed orbit is the disjoint union of the orbit itself together with orbits of strictly smaller dimensions. Thus any algebraic automorphism of $\mathcal{O}$ fixes (shuffles) orbits if and only if it fixes (shuffles) closures of orbits.

According to [1, p. 97] $S_{t}\left(D_{p}\right)$ is algebraic as it is the stabilizer of the closed set $D_{p}$. And since $\Lambda^{p} G$ is connected algebraic, $\mathfrak{F}_{1}$ and $\mathcal{S}_{1}$ are are algebraic as intersections of transporters of closed sets (i.e. closures of orbits). Also $A\left(G_{p}\right), \mathcal{F}$, and $\mathcal{S}$ are algebraic since they are images of $\mathrm{St}\left(D_{p}\right), \mathcal{F}_{1}$, and $\mathcal{S}_{1}$ by the rational homomorphism $\pi$. According to Westwick's theorem $\left(\mathcal{S}_{1}: \Lambda^{p \mathcal{S}_{2}}\right) \leq 2,\left(\mathcal{F}_{1}: \Lambda^{p \mathcal{F}_{2}}\right) \leq 2$. Thus $\Lambda^{p \mathcal{S}_{2}}$ and $\Lambda^{p \mathcal{F}_{2}}$ are closed (algebraic) and their inverse images $\mathcal{S}_{2}$ and $\mathcal{F}_{2}$ are closed in $G L(V)$, hence algebraic.

Notation. For any algebraic group $H$ we let $L(H)$ be its Lie algebra.

Proposition 2. (1) $L\left(\mathfrak{F}_{2}\right)$ is an ideal in $L\left(\mathcal{S}_{2}\right)$.

(2) $(\mathcal{S}: \mathfrak{F}) \leq 2\left(\mathcal{S}_{2}: \mathcal{F}_{2}\right)^{2}$. 
Proof. (1) $\mathcal{S}$ normalizes $\mathcal{F}$ and $\pi$ is always surjective so $\mathcal{S}_{1}$ normalizes $\mathcal{F}_{1}$. Thus $L\left(\mathcal{S}_{1}\right)$ normalizes $L\left(\mathcal{F}_{1}\right)$. At worst $\left(\operatorname{St}_{p}\left(D_{p}\right): \Lambda^{p}(G L(V))\right)=2$, hence $\left(\mathcal{S}_{1}: \Lambda^{p \mathcal{S}_{2}}\right) \leq 2,\left(\mathcal{F}_{1}: \Lambda^{p \mathcal{F}_{2}}\right) \leq 2$. Therefore $d \Lambda^{p}$ (the differential) maps $L\left(\mathcal{S}_{2}\right)$ onto $L\left(\mathcal{S}_{1}\right)$ and $L\left(\mathcal{F}_{2}\right)$ onto $L\left(\mathcal{F}_{1}\right)$. As a result $L\left(\mathcal{S}_{2}\right)$ normalizes $L\left(\mathcal{F}_{2}\right)$.

(2) Since $\pi$ is surjective $(\mathcal{S}: \mathcal{F}) \leq\left(\mathcal{S}_{1}: \mathcal{F}_{1}\right)$. Also, since $\left(\mathcal{S}_{1}: \Lambda^{p \mathcal{S}_{2}}\right) \leq 2$, $\left(\mathcal{F}_{1}: \Lambda^{p F_{2}}\right) \leq 2$, one easily sees that

$$
\left(\mathcal{S}_{1}: \mathcal{F}_{1}\right) \leq 2\left(\Lambda^{p} \mathcal{S}_{2}: \Lambda^{p F_{F}}\right) \leq 2\left(\mathcal{S}_{2}: \mathscr{F}_{2}\right)
$$

Theorem 3. Suppose $G$ is a connected, irreducible algebraic subgroup of $\mathrm{GL}(V)$ containing the nonzero scalars. Let $\mathcal{S}, \mathcal{F}$ be the orbit shuffling and fixing groups for the induced action of $G$ on the projective Grassmann variety of all p-dimensional subspaces of $V$. Then $\mathcal{S} / \mathcal{F}$ is a finite group.

Proof. By (2) of the proposition it suffices to show that $\mathcal{S}_{2} / \mathcal{F}_{2}$ is a finite group. Since $\mathcal{S}_{2}$ and $\mathcal{F}_{2}$ are algebraic groups, it suffices to show $L\left(\mathcal{S}_{2}\right)=L\left(\mathfrak{F}_{2}\right)$.

Since $G$ acts irreducibly on $V$ so does $L(G)$. In fact $L\left(\mathcal{F}_{2}\right)$ acts irreducibly since it contains $L(G)$. Let $L=L\left(\mathcal{F}_{2}\right)$. Then $L=[L, L] \oplus$ $Z(L)(Z(L)=$ center of $L),[L, L]$ is semisimple, and $Z(L)=K I_{V}$ since $L$ acts irreducibly. By part (1) of the proposition, $L\left(\delta_{2}\right)$ normalizes $L=$ $[L, L] \oplus K I_{V}$, hence it normalizes $[L, L]$. Since $[L, L]$ is a semisimple ideal in $L\left(\mathcal{S}_{2}\right), L\left(\mathcal{S}_{2}\right)$ splits as the direct sum of $[L, L]$ with its centralizer in $L\left(\mathcal{S}_{2}\right)$. Since $L$ acts irreducibly and $L=[L, L] \oplus K I_{V},[L, L]$ also acts irreducibly. Thus its centralizer in $L\left(\delta_{2}\right)$ must consist only of scalars. Hence $L\left(\mathcal{S}_{2}\right)=[L, L] \oplus K I_{V}=L$.

\section{REFERENCES}

1. A. Borel, Linear algebraic groups, Benjamin, New York, 1969. MR 40 \#4273.

2. M. Gauger, Duality theories for metabelian Lie algebras, Trans. Amer. Math. Soc. 187 (1974), 89-102.

3. - Duality theories for metabelian Lie algebras. II, Trans. Amer. Math. Soc. (to appear).

4. R. Westwick, Linear transformations on Grassmann spaces, Pacific J. Math. 14 (1964), 1123-1127. MR $29 \# 4766$.

DEPARTMENT OF MATHEMATICS AND STATISTICS, UNIVERSITY OF MASSACHUSETTS, AMHERST, MASSACHUSETTS 01002 